Article

\title{
Numerical Analysis of the Magnetic Field of High-Current Busducts and GIL Systems
}

\section{Petar Sarajcev}

University of Split, Faculty of Electrical Engineering Mechanical Engineering and Naval Architecture, R. Boskovica 32, HR-21000 Split, Croatia; E-Mail: petar.sarajcev@fesb.hr; Tel.: +385-21-305-806; Fax: +385-21-305-776

Received: 25 October 2011; in revised form: 29 November 2011 / Accepted: 5 December 2011 / Published: 13 December 2011

\begin{abstract}
This paper presents a numerical computation method for determining the magnetic field of high-current busducts of circular cross-section geometry, based on the subdivision of the busduct phase conductors and screens into the conductor filaments and the subsequent application of the mesh-current method, with the aid of the geometric mean distance method. The mathematical model takes into account the skin effect and the proximity effects, as well as the complete electromagnetic coupling between phase conductors and metal enclosures (i.e., screens) of the single-phase isolated busduct system (of circular cross-section geometry). This model could be readily applied to the computation of the magnetic field of the Gas Insulated Transmission Lines (GIL) as well.
\end{abstract}

Keywords: high-current busduct; gas-insulated transmission line; electromagnetic field; filament method; mesh-current method; geometric mean distance method

\section{Introduction}

High-current busduct systems are usually air insulated single-phase enclosure systems with tubular aluminum or copper conductors encapsulated in the coaxial aluminum enclosure (i.e., screen). Hence, they are circular in cross-section geometry, laid in horizontal or vertical three-phase formation. Their main function is seen in connecting generators with their step-up transformers. Due to the medium voltage level of the generator terminals $(10 \mathrm{kV}$ to $30 \mathrm{kV})$ and very high current output, usually in the range of $5 \mathrm{kA}$ up to even $30 \mathrm{kA}$, high-current busducts are seen as electric power transmission lines 
facilitating very efficient way of evacuating power from the generator units, especially in case of, e.g., hydro power plants, where generator units are housed in a cavern [1-3].

Gas-insulated transmission lines (GIL) represent a high voltage electric power transmission technology suitable for very efficient transmission of very high volumes of electric power across both short and long distances [4-6]. They could be operated with rated voltages ranging from $123 \mathrm{kV}$ to $550 \mathrm{kV}$ and rated currents in excess of $4500 \mathrm{~A}$. The insulating media is usually a mixture of nitrogen (80\%) and sulphur-hexafluoride (20\%) gases, i.e., a $\mathrm{N}_{2}-\mathrm{SF}_{6}$ gas mixture. The insulating media, thus, facilitates the geometry of the GIL system being very similar (in terms of the cross-section geometry) to that of the high-current busducts. It features tubular aluminum phase conductors in single-phase isolated coaxial aluminum screens. Hence, mathematical models of the high-current busducts are directly applicable to the analysis of GIL systems, allowing for the small differences in manufacturing details.

The GIL technology is increasingly becoming very popular in bulk transmission of high volumes of electric power (over short and long distances), with several major projects in Europe and China either being in the planning phase or already in operation, e.g., [4,7-10]. This expansion of the GIL technology application will probably accelerate in the near future, e.g., [11]. GIL systems could be laid on the earth's surface, buried in the earth or installed in tunnels, e.g., in existing railway tunnels (either in horizontal or vertical configuration), $[4,8]$. The technology from the oil pipelines construction has been adopted for the purpose. The GIL technology has several very promising features, such as: low transmission losses, low capacitive load, power rating of the equivalent overhead transmission line, high reliability, no electric ageing, no thermal ageing, operation equal to that of the overhead transmission line (i.e., auto-reclosure of the distance protection function), low environmental influence, etc. All of these beneficial factors will surely add to its increased application in the near future, e.g., particularly for bringing the bulk transmission power to major city centers.

High-current busducts and/or GIL systems, as just mentioned, usually have aluminum phase conductors encapsulated in an aluminum enclosure of circular cross-section geometry. The screens of the high-current busducts, which are generally up to several hundred meters long, could be grounded on a single end or on both ends (they could possibly be additionally grounded on several places along the route). With GIL systems, screens are continuously grounded along the transmission line route, which could span several kilometers or more, e.g., [4,6]. Additionally, screens of different phases are mutually bonded (i.e., short-circuited with appropriate current-carrying conductors) along the route, both for the high-current busducts and GIL systems. This establishes the current paths for the so-called circulating currents (along with eddy currents) in the screens, which oppose the phase currents inducing them through the strong electromagnetic coupling (additionally influenced by the skin and proximity effects), e.g., [12-14]. Both phase conductor current and (induced) screen current distribution generate external electromagnetic field, e.g., [13,14]. This electromagnetic field could have adverse effects on neighboring electrical equipment or present undesirable environmental aspects regarding public concerns of possible harmful effects caused by human exposure to extremely low frequency fields.

This paper presents an efficient numerical method for determining the magnetic field of high-current busducts and/or GIL systems, based on the conductor filament method, with application of the mesh-current method, aided by the geometric mean distance method, e.g., [12]. Proposed approach accounts for the skin effect and the proximity effects, as well as the complete electromagnetic coupling 
between phase conductors and metal enclosures of the single-phase isolated busduct or GIL system of circular cross-section geometry. This approach differs in terms of the problem domain decomposition from the finite element method (FEM) approach, which has been seen as a standard method of approach in dealing with this category of electromagnetic problems, and has been implemented in several commercially available software packages, e.g., [15]. Here presented method of approach is more efficient then the FEM procedure, in treating the busduct and/or GIL systems, due to the fact that the FEM approach - in this open boundary problem - needs to carry-out discretization of the very large solution region, e.g., [15]. On the other hand, filament method only needs the discretization of the busduct system itself (i.e., its cross-sectional geometry), thus resulting in a far lower number of (finite) elements (i.e., filaments). Finally, it ought to be mentioned that this magnetic field problem could be treated in the purely analytical manner, as described in, e.g., [13,16,17].

\section{Mathematical Model}

Figure 1 graphically depicts the high-current busduct (or GIL system), with screens mutually bonded and grounded on both ends. The treatment of the operating conditions for the busduct or GIL system is also illustrated in this figure, as will be elaborated upon later in the paper.

Figure 1. Graphical depiction of the high-current busduct (or GIL system) with screens mutually bonded and grounded on both ends.

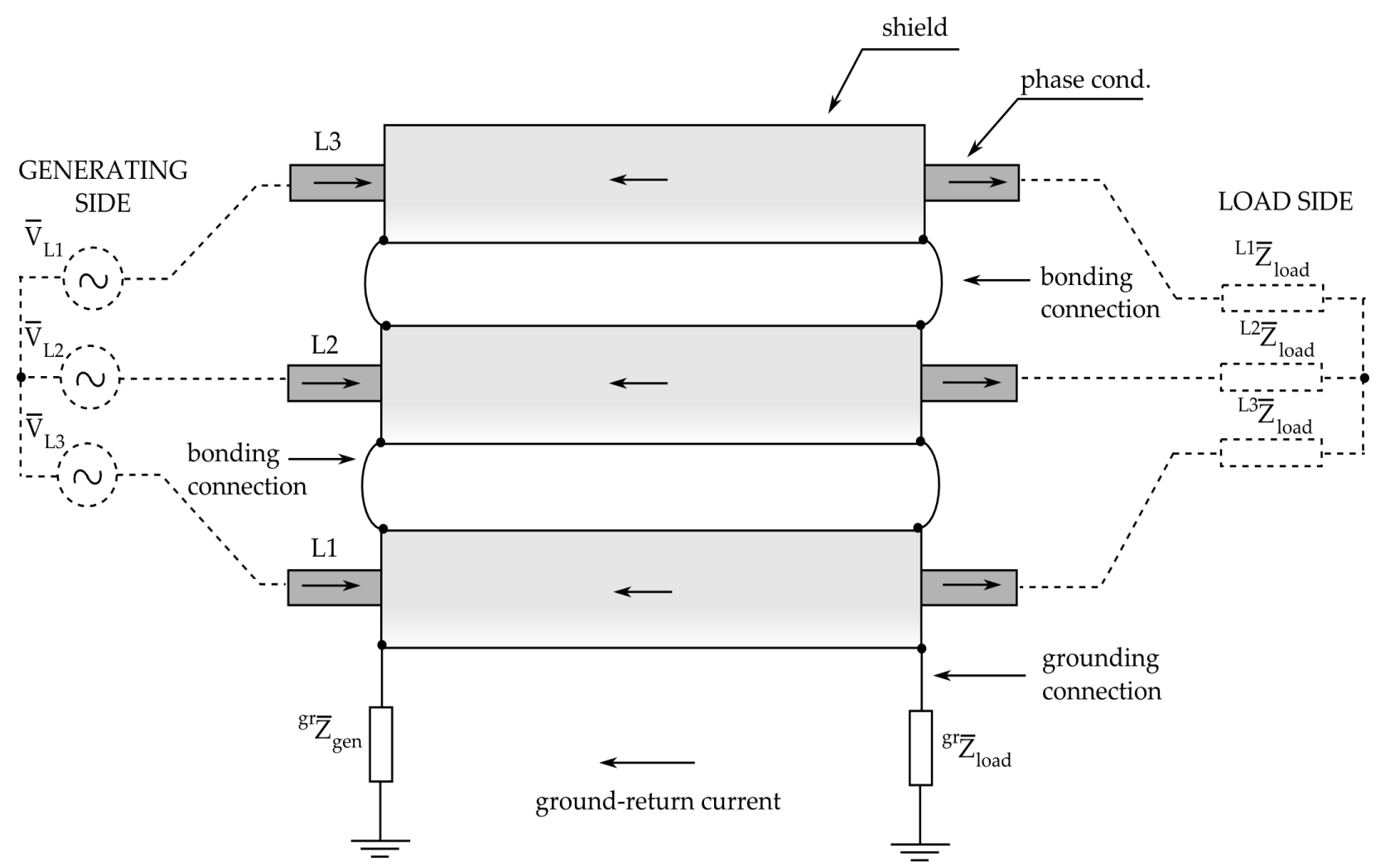

The basis for the efficient numerical computation of high-current busduct or GIL system electromagnetic fields emanates from a mathematical model for obtaining the exact current distribution in the phase conductors and screens of the system at hand, which should account for the skin and proximity effects. This model is in turn based on the application of the filament method, where phase 
conductors and screens are replaced by a number of smaller subconductors or filaments. The following assumptions are introduced: (i) the current flows only longitudinally in the filament; (ii) the material resistivity and permeability are uniform within each filament and independent of current, but they may differ from those of other filaments; (iii) all filaments are parallel. The skin and proximity effects are directly incorporated within the proposed filament method.

This method has been already successfully applied to high voltage cables in [18,19] and to a high-current busduct of rectangular cross-section geometry in [12]. The method itself is well documented and explained in [12] and will not be repeated here. It should be mentioned that each phase conductor is divided into Nc filaments, while each screen is divided into Ns filaments; coordinates of the filaments are determined in regards to the global coordinate system (positioned arbitrarily). Hence, the total number of filaments equals $\mathrm{N}=3 \mathrm{Nc}+3 \mathrm{Ns}$. The mentioned numbers of filaments for the phase conductors and screens are chosen in such a manner that the current density remains uniform across the surface (of each) of the filaments; this necessitates a rather large number of filaments.

A mesh-current method is applied in order to determine the unknown conductor filament currents, e.g., $[12,18]$. Each mesh is represented by a loop consisting of: (1) the associated phase conductor or screen filament; (2) the associated voltage and the load impedance of the phase, if the loop contains the phase conductor filament and (3) the ground return path. Moreover, governing equations are formulated using the additional assumptions: elbows (if exist) of the busduct or GIL system are neglected and the transmission line is longitudinally homogenous. Thus a following general system of complex linear algebraic equations could be formed [12]:

$$
\{\mathbf{V}\}=[\mathbf{Z}] \cdot\{\mathbf{I}\}
$$

where $\{\mathbf{V}\}$ - known vector of the loop voltages, defined by the conditions at the generating-side terminals; $[\mathbf{Z}]$-matrix of self and mutual impedances of the loops; this matrix is complex and symmetric in respect to its main diagonal (but not Hermitian), $\{\mathbf{I}\}$ - unknown vector of the filament currents.

In the process of deriving the self and mutual impedances of the loops (created by filaments as explained above) one firstly needs to compute the associated distances between the involved filaments. Here a geometric mean distance (GMD) method is employed, accounting for the fact that the filaments are rectangular in cross-section, [12]. For the arbitrary $i$-th filament of a rectangular cross section, self GMD is given by [12]:

$$
d_{i i}=0.2235 \cdot\left(a_{i}+b_{i}\right)
$$

where $a_{i}$ and $b_{i}$ represent the dimensions of the filament cross-section. Also, GMD between any two filaments of the busduct system is given as a geometrical distance between their central points, i.e., the GMD between the $i$-th and the $k$-th filament is given by:

$$
d_{i k}=\sqrt{\left(x_{i}-x_{k}\right)^{2}+\left(y_{i}-y_{k}\right)^{2}}
$$

where $x_{i}, y_{i}$ represent coordinates of $i$-th filament center, while $x_{k}, y_{k}$ represent coordinates of the $k$-th filament center.

The system of equations given by (1), in case of the busduct or GIL system screens being grounded on both ends and mutually bonded, could be transformed into the following: 


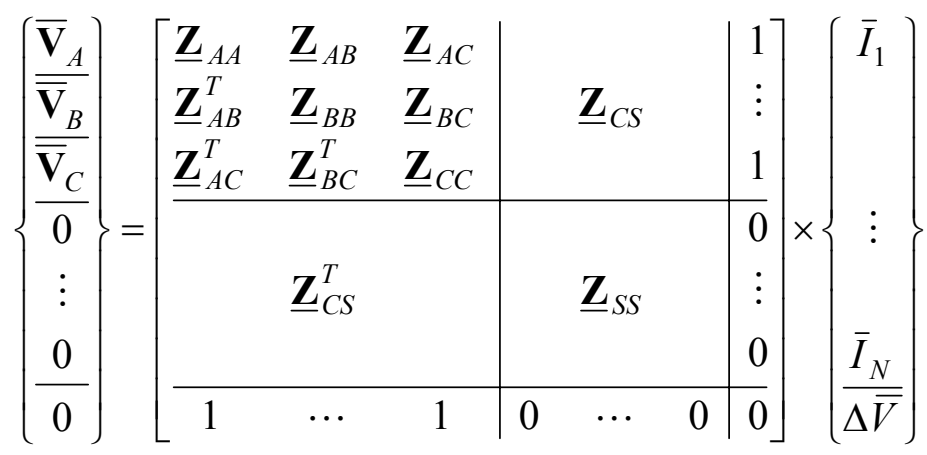

with:

$$
\begin{aligned}
& \overline{\mathbf{V}}_{A}=\{1\}^{T} \cdot \bar{V}_{L 1} \\
& \overline{\mathbf{V}}_{B}=\{1\}^{T} \cdot \bar{V}_{L 2} \\
& \overline{\mathbf{V}}_{C}=\{1\}^{T} \cdot \bar{V}_{L 3}
\end{aligned}
$$

where $\{1\}^{T}$ is a transposed all-ones vector having Nc elements. Here, $\bar{V}_{L 1}, \bar{V}_{L 2}$ and $\bar{V}_{L 3}$ represent phase voltages on the so-called generating side of the busduct or GIL system, in phases L1, L2 and L3, which could have arbitrarily selected input values (observe Figure 1).

The matrix of self and mutual impedances of the filament loops in Equation (4) consists of several submatrices; their description is as follows: $\underline{\mathbf{Z}}_{A A}, \underline{\mathbf{Z}}_{B B}, \underline{\mathbf{Z}}_{C C}$-square submatrices each with dimension (Nc, Nc) of self and mutual impedances of the loops involving filaments, respectively, of the phase conductors L1, L2 and L3; $\underline{\mathbf{Z}}_{A B}, \underline{\mathbf{Z}}_{A C}, \underline{\mathbf{Z}}_{B C}$-square submatrices each with dimension (Nc, Nc) of mutual impedances of the loops involving phase conductors, respectively, of the phases L1-L2, L1-L3 and L2-L3; same can be said for their transposed submatrices (denoted with superscript T); $\underline{\mathbf{Z}}_{C S}$-rectangular submatrix with dimension ( $3 \mathrm{Nc}, 3 \mathrm{Ns}$ ) of mutual impedances between the loops involving filaments of all phase conductors and all the screens; $\underline{\mathbf{Z}}_{S S}$-square submatrix with dimension (3Ns, 3Ns) of self and mutual impedances of the loops involving filaments of the screens (for the phases L1, L2 and L3).

The above mentioned self and mutual impedances (including the ground return path) for the loops involving filaments of the phase conductors and screens, as well as the various mutual impedances involved, are computed on the basis of the well-known Carson theory (simple closed-form approximations for the overhead wires in the low-frequency range are used), see e.g., [12,18,20,21]. It should be mentioned that the resistance of each of the filaments involved is taken as a function of the operating temperature; phase conductors and screens will have different operating temperatures (although they might be of the same material), [12]. The mathematical expressions needed for computing the aforementioned impedances are provided in [12]. Additionally, inclusion of the terminal conditions on the so-called generating and the load sides of the high-current busduct (or GIL system) have been explained in detail in [12] and, thus, will not be repeated here. It could be stated that the busduct generating side is represented with the ideal three-phase voltage source, while the load side is represented with a three-phase burden impedances (observe Figure 1), [12,18]. Hence, the various operating conditions of the high-current busduct (or GIL system) could be simulated in this way, from normal operation, to unbalanced loads, to various possible short circuit events. 
Having said all of the above, while considering the notations in the Figure 1, self and mutual impedances of submatrices $\underline{\mathbf{Z}}_{A A}, \underline{\mathbf{Z}}_{B B}, \underline{\mathbf{Z}}_{C C}$ could be determined from the following expressions:

$$
\begin{aligned}
& \bar{Z}_{i i}={ }^{c a r} \bar{Z}_{i i}+{ }^{\alpha} \bar{Z}_{\text {load }} \\
& \bar{Z}_{i k}={ }^{c a r} \bar{Z}_{i k}+{ }^{\alpha} \bar{Z}_{\text {load }}
\end{aligned}
$$

where $\alpha$ takes on the reference to phases L1, L2 and L3, as appropriate. The impedances ${ }^{c a r} \bar{Z}_{i i}$ and ${ }^{c a r} \bar{Z}_{i k}$ in the above expressions represent simple closed-form Carson's expressions for the self and mutual filament impedances, including the ground return path, [12,18]. Also, self and mutual impedances of the submatrix $\underline{\mathbf{Z}}_{S S}$, considering the Figure 1, are determined as follows:

$$
\begin{aligned}
& \bar{Z}_{i i}={ }^{c a r} \bar{Z}_{i i}+{ }^{g r} \bar{Z}_{\text {gen }}+{ }^{g r} \bar{Z}_{\text {load }} \\
& \bar{Z}_{i k}={ }^{c a r} \bar{Z}_{i k}+{ }^{g r} \bar{Z}_{\text {gen }}+{ }^{g r} \bar{Z}_{\text {load }}
\end{aligned}
$$

where newly introduced impedances ${ }^{g r} \bar{Z}_{\text {gen }}$ and ${ }^{g r} \bar{Z}_{\text {load }}$ represent the equivalent impedances of the grounding systems at the generating and load sides of the busduct system. Finally, all other impedances found in the matrix Equation (4) could be determined from the following expression:

$$
\bar{Z}_{i k}={ }^{c a r} \bar{Z}_{i k}
$$

The unknown filament currents for the phase conductors and screens become known once the system of complex linear algebraic equations in (4) is solved (involving here $\mathrm{N}+1$ equations with that many unknowns; i.e., $3 \mathrm{Nc}+3 \mathrm{Ns}$ unknown filament currents and an unknown voltage difference $\Delta \bar{V}$ between generating and load busduct side). This is a rather straightforward task. With all the filament currents known, determining the equivalent currents of the phase conductors and screens becomes a matter of programmer's book-keeping, i.e., tracking the position of each filament within the system and summating current contributions from all filaments which are part of the conductor (or screen) at hand; see [12] for more details. Furthermore, with all of the filament currents known, Joule power losses of the bus ducts are easily obtained, again through the programmer's book-keeping of sorts, as described in [12]. Computation of the power losses takes into account, not only the actual current distribution in the bus ducts (which includes the skin and proximity effects), but the temperature-dependent resistance of the material as well.

The determined distribution of currents in phase conductors and screens of the busduct or GIL system (i.e., filament currents), influenced themselves by skin and proximity effects, gives rise to the electromagnetic field. In order to determine the field distribution (i.e., magnetic field outside of the high-current busduct or GIL system), let us introduce geometry provided in the Figure 2. It has been assumed in this figure, without the loss of generality, that the magnetic field intensity will be computed in the points of the observational circle arbitrarily positioned in respect to the busduct (or GIL system) at hand. Field points are, thus, positioned in air (or even in earth) which surrounds the high-current busduct or GIL system (i.e., in the non-magnetic material). Consequently, Figure 2 depicts a geometry involved in computing the magnetic field at a single point, i.e., so-called field point $\mathrm{M}_{\mathrm{k}}\left(x_{k}, y_{k}\right)$ due to the current of the single filament, i.e., source point $\mathrm{T}_{\mathrm{i}}\left(x_{i}, y_{i}\right)$, which could belong either to the phase conductor or to the screen. Both points have been designated with their global coordinates. It can be 
observed from the Figure 2 that the magnetic field intensity at the field point is composed of two (mutually perpendicular) partial field components; i.e., the radial and the tangential field component at the field point (on the arbitrarily positioned observational circle).

Figure 2. Geometry involved in determining the magnetic field due to current distribution in high-current busduct.

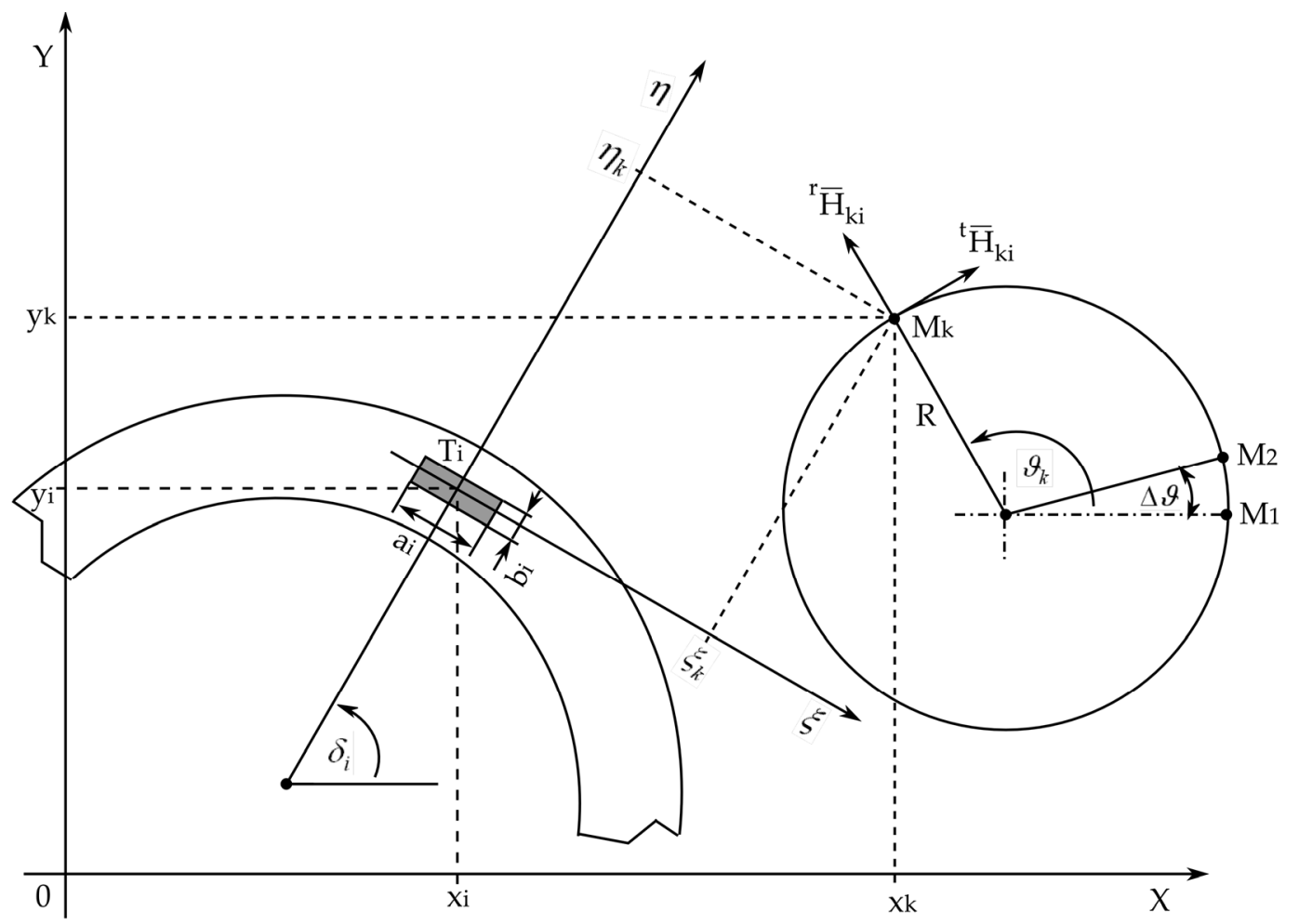

According to the notation introduced in the Figure 2, local coordinates of the field point, i.e., $\mathrm{M}_{\mathrm{k}}\left(\xi_{k}, \eta_{k}\right)$ could be easily obtained from the introduced global coordinates of the field and source points, as follows (local coordinate system is positioned at the source point, i.e., at the center of the filament at hand):

$$
\begin{gathered}
\xi_{k}=\left(x_{k}-x_{i}\right) \cdot \sin \delta_{i}-\left(y_{k}-y_{i}\right) \cdot \cos \delta_{i} \\
\eta_{k}=\left(x_{k}-x_{i}\right) \cdot \cos \delta_{i}+\left(y_{k}-y_{i}\right) \cdot \sin \delta_{i}
\end{gathered}
$$

Furthermore, partial tangential and radial components of the magnetic field intensity phasors at the arbitrary field point $\mathrm{M}_{\mathrm{k}}$, due to the filament current at the source point $T_{i}$, could be respectively obtained from the following expressions:

$$
\begin{aligned}
{ }^{t} \underline{H}_{k i} & ={ }^{\xi} \underline{H}_{k i} \cdot \cos \left(\vartheta_{k}-\delta_{i}\right)+{ }^{\eta} \underline{H}_{k i} \cdot \sin \left(\vartheta_{k}-\delta_{i}\right) \\
{ }^{r} \underline{H}_{k i} & =-{ }^{\xi} \underline{H}_{k i} \cdot \sin \left(\vartheta_{k}-\delta_{i}\right)+{ }^{\eta} \underline{H}_{k i} \cdot \cos \left(\vartheta_{k}-\delta_{i}\right)
\end{aligned}
$$

where ${ }^{\xi} \bar{H}_{k i}$ and ${ }^{\eta} \bar{H}_{k i}$ represent partial components of the magnetic field intensity phasors along the local coordinate axes $\xi$ and $\eta$, respectively. From the well-known application of the Biot-Savart law on the geometry provided in the Figure 2 (i.e., magnetic field in the vicinity of the rectangular 
current-carrying filament of "infinite" length), partial component of the magnetic field intensity phasor along the local coordinate axis $\xi$ could be computed as follows, e.g., [22,23]:

$$
\begin{aligned}
& { }^{\xi} \bar{H}_{k i}=-\frac{\bar{I}_{i}}{2 \pi \cdot a_{i} \cdot b_{i}} \cdot\left[\frac{1}{2} \cdot\left(\xi_{k}+\frac{a_{i}}{2}\right) \cdot \ln \frac{\left(\eta_{k}+\frac{b_{i}}{2}\right)^{2}+\left(\xi_{k}+\frac{a_{i}}{2}\right)^{2}}{\left(\eta_{k}-\frac{b_{i}}{2}\right)^{2}+\left(\xi_{k}+\frac{a_{i}}{2}\right)^{2}}-\right. \\
& -\frac{1}{2} \cdot\left(\xi_{k}-+\frac{a_{i}}{2}\right) \cdot \ln \frac{\left(\eta_{k}+\frac{b_{i}}{2}\right)^{2}+\left(\xi_{k}-\frac{a_{i}}{2}\right)^{2}}{\left(\eta_{k}-\frac{b_{i}}{2}\right)^{2}+\left(\xi_{k}-\frac{a_{i}}{2}\right)^{2}}+ \\
& \left.+\left(\eta_{k}+\frac{b_{i}}{2}\right) \cdot\left(a \tan \frac{\xi_{k}+\frac{a_{i}}{2}}{\eta_{k}+\frac{b_{i}}{2}}-a \tan \frac{\xi_{k}-\frac{a_{i}}{2}}{\eta_{k}+\frac{b_{i}}{2}}\right)^{2}-\left(\eta_{k}-\frac{b_{i}}{2}\right) \cdot\left(a \tan \frac{\xi_{k}+\frac{a_{i}}{2}}{\eta_{k}-\frac{b_{i}}{2}}-a \tan \frac{\xi_{k}-\frac{a_{i}}{2}}{\eta_{k}-\frac{b_{i}}{2}}\right)\right]
\end{aligned}
$$

By the way of analogy, the partial component of the magnetic field intensity phasor along the local coordinate axis $\eta$ is determined from the following expression:

$$
\begin{aligned}
& { }^{\eta} \bar{H}_{k i}=\frac{\bar{I}_{i}}{2 \pi \cdot a_{i} \cdot b_{i}} \cdot\left[\frac{1}{2} \cdot\left(\eta_{k}+\frac{b_{i}}{2}\right) \cdot \ln \frac{\left(\xi_{k}+\frac{a_{i}}{2}\right)^{2}+\left(\eta_{k}+\frac{b_{i}}{2}\right)^{2}}{\left(\xi_{k}-\frac{a_{i}}{2}\right)^{2}+\left(\eta_{k}+\frac{b_{i}}{2}\right)^{2}}-\right. \\
& -\frac{1}{2} \cdot\left(\eta_{k}-\frac{b_{i}}{2}\right) \cdot \ln \frac{\left(\xi_{k}+\frac{a_{i}}{2}\right)^{2}+\left(\eta_{k}-\frac{b_{i}}{2}\right)^{2}}{\left(\xi_{k}-\frac{a_{i}}{2}\right)^{2}+\left(\eta_{k}-\frac{b_{i}}{2}\right)^{2}}+ \\
& \left.+\left(\xi_{k}+\frac{a_{i}}{2}\right) \cdot\left(a \tan \frac{\eta_{k}+\frac{b_{i}}{2}}{\xi_{k}+\frac{a_{i}}{2}}-a \tan \frac{\eta_{k}-\frac{b_{i}}{2}}{\xi_{k}+\frac{a_{i}}{2}}\right)-\left(\xi_{k}-\frac{a_{i}}{2}\right) \cdot\left(a \tan \frac{\eta_{k}+\frac{b_{i}}{2}}{\xi_{k}-\frac{a_{i}}{2}}-a \tan \frac{\eta_{k}-\frac{b_{i}}{2}}{\xi_{k}-\frac{a_{i}}{2}}\right)\right]
\end{aligned}
$$

The total tangential and radial components of the magnetic field intensity phasors at the field point $\mathrm{M}_{\mathrm{k}}\left(x_{k}, y_{k}\right)$ are obtained by a superposition, due to the influence of all the filament currents (of all the phase conductors and all the screens), as follows:

$$
\begin{aligned}
& { }^{t} \bar{H}_{k}=\sum_{i=1}^{N}{ }^{t} \bar{H}_{k i} \\
& { }^{r} \bar{H}_{k}=\sum_{i=1}^{N}{ }^{r} \bar{H}_{k i}
\end{aligned}
$$

Ampere's circuit law could be invoked here in order to show that the phasor of the magnetic field intensity at the field points of the observational circle have been computed properly; this has been in-fact accomplished in the associated computer program. Finally, the RMS value of the total magnetic 
field intensity at the field point is obtained from the previously computed total tangential and radial components, according to the well-known expression:

$$
H_{k}=\sqrt{\left|t \bar{H}_{k}\right|^{2}+\left|{ }^{r} \bar{H}_{k}\right|^{2}}
$$

The appropriate value of the total magnetic flux density at the field point is simply obtained from the above determined total magnetic field intensity, by using the following relation:

$$
B_{k}=\mu_{0} \cdot H_{k}
$$

with $\mu_{0}=4 \pi \cdot 10^{-7} \mathrm{Vs} / \mathrm{Am}$. The above presented procedure is repeated for each of the field points, which form the observational circle. A similar treatment is implemented for the field points positioned along the observational profiles.

\section{Numerical Example}

Figure 3 graphically depicts the cross-section of the high-current busduct under consideration. Dimensions are given in millimeters.

Figure 3. Graphical depiction of the cross-section geometry of the high-current busduct under consideration.

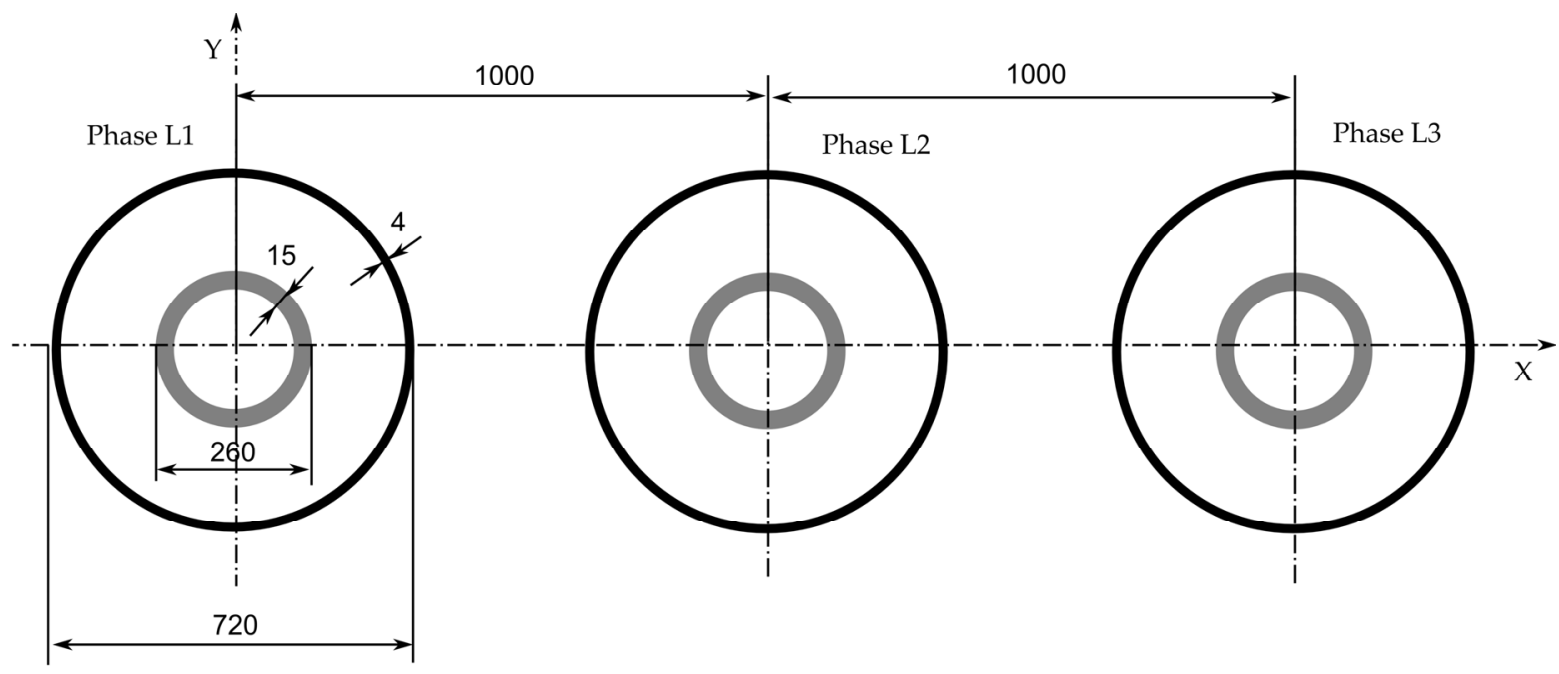

Phase conductors and screens of the busduct are assumed to be made of high-quality aluminum (Al 99.5) with conductivity of $34.2 \mathrm{MS} / \mathrm{m}$ at $20{ }^{\circ} \mathrm{C}$, e.g., [3]. Maximum allowable operating over-temperature of the phase conductors is assumed to be $65^{\circ} \mathrm{C}$, while that of the screen equals $40{ }^{\circ} \mathrm{C}$, above the temperature of the surrounding medium (i.e., air) which is given as $40^{\circ} \mathrm{C}$. Rated voltage of the busduct system is assumed to be $24 \mathrm{kV}$ (phase-to-phase) with rated continuous operating current of 7000 A. Length of the busduct system is assumed to be $100 \mathrm{~m}$. For the relative soil resistivity of the site a value of $100 \Omega \mathrm{m}$ is provided. Impedances of the grounding systems at the generating and load sides of the busduct are neglected here (i.e., taken equal to zero). Finally, it is assumed that the treated busduct system carries its rated (nominal) operating currents at rated voltage (three-phase symmetrical operating condition). 
According to the procedure for the numerical solution of the filament current distribution, each phase conductor of the busducts (viewed in cross-section) is subdivided into 500 filaments (three concentric layers of equal thickness; first two layers having 150 filaments each, while the third one having 200 filaments). Additionally, each screen of the high-current busduct is subdivided into 400 filaments, giving a total of 2700 filaments. This particular division into filaments is carried-out, as previously mentioned, in such a manner that the cross section for each of the filaments is kept small enough so that the surface current density (on its cross-section) remains uniform.

In order to depict the distribution of the magnetic field in the vicinity of the high-current busduct at hand, an observational circle is positioned (10 cm above the screen's surface) outside each of the phases of the busduct system. Each of the observational circles contains 3000 field points. Additionally two observational profiles are also provided; first at $0.5 \mathrm{~m}$ and the second at $1 \mathrm{~m}$ above the horizontal symmetry line of the high-current busduct system (which is itself positioned horizontally in respect to the earth surface). Observational profiles extend $1 \mathrm{~m}$ to the left and right of the busduct system. Each observational profile contains 4000 field points.

Table 1 presents results of the numerical computation of the current distribution in the phase conductors and screens of the high-current busduct. It can be nicely observed from this table that the screen currents are almost equal to the phase currents (even larger in one of the phases), due to the fact that there are current paths, formed by the screen bonding, giving rise to the so-called circulating currents. There is also the earth-return current, circulating between the grounding systems located on both sides of the busduct route.

Table 1. Current distribution in the high-current busducts (screens grounded and mutually bonded on both ends and possibly additionally along the route).

\begin{tabular}{cccc}
\hline \multirow{2}{*}{ Currents } & \multicolumn{3}{c}{ I (A) $\angle\left(^{\circ}\right)$} \\
\cline { 2 - 4 } & Phase L1 & Phase L2 & Phase L3 \\
\hline Phase conductors & $6997 \angle-0.3^{\circ}$ & $6997 \angle 239.7^{\circ}$ & $6997 \angle 119.7^{\circ}$ \\
Screens & $6847 \angle 182.8^{\circ}$ & $6977 \angle 64.7^{\circ}$ & $7122 \angle-57.1^{\circ}$ \\
Earth & & $12.1 \angle-24^{\circ}$ & \\
\hline
\end{tabular}

It is quite evident from the data presented in this table that the screen currents do not form a symmetric three-phase system; there is an asymmetry present among the screen currents, which is due to the inherent electromagnetic asymmetry found in the flat formation of the three-phase conductor arrangements, e.g., [22]. It has been found that the largest screen current will be induced in that particular screen of which the associated phase conductor carries the leggiest phase (i.e., phase L3).

Table 2 presents numerically computed Joule power losses of the high-current busduct, relevant for the proposed operating condition and screen treatment, including the operating temperatures of the phase conductors and screens. According to the analysis carried out with the commercial software package MagNet, [15], the total busduct power losses of $1088 \mathrm{~W} / \mathrm{m}$ have been obtained. Comparison of this result with that form the table 2 yields a relative difference of $1.47 \%$, which is mainly due to the differences in treating, i.e., imposing the busduct operating conditions (as well as some minor differences in the busduct geometry), [24]. 
Table 2. Computed power losses of the high-current bus duct (screens grounded and mutually bonded on both ends and possibly additionally along the route).

\begin{tabular}{cccc}
\hline \multirow{2}{*}{ Power Losses } & \multicolumn{3}{c}{ P $(\mathbf{W} / \mathbf{m})$} \\
\cline { 2 - 4 } & Phase L1 & Phase L2 & Phase L3 \\
\hline Phase conductors & 173 & 173 & 173 \\
Screens & 177 & 184 & 192 \\
Total & & 1072 & \\
\hline
\end{tabular}

Furthermore, Figure 4 graphically depicts the total magnetic field distribution along the three observational circles (each positioned $10 \mathrm{~cm}$ above the screen) outside each of the three phases of the high-current busduct system. This figure in-fact provides the RMS values of the total magnetic flux density at the field points on the three observational circles, depicted as polar plots, with local coordinates of field points for each of the circles (see also Figure 2).

Figure 4. Distribution of the RMS values of the total magnetic flux density at the field points of the observational circles positioned outside each of the phases of the busduct system.
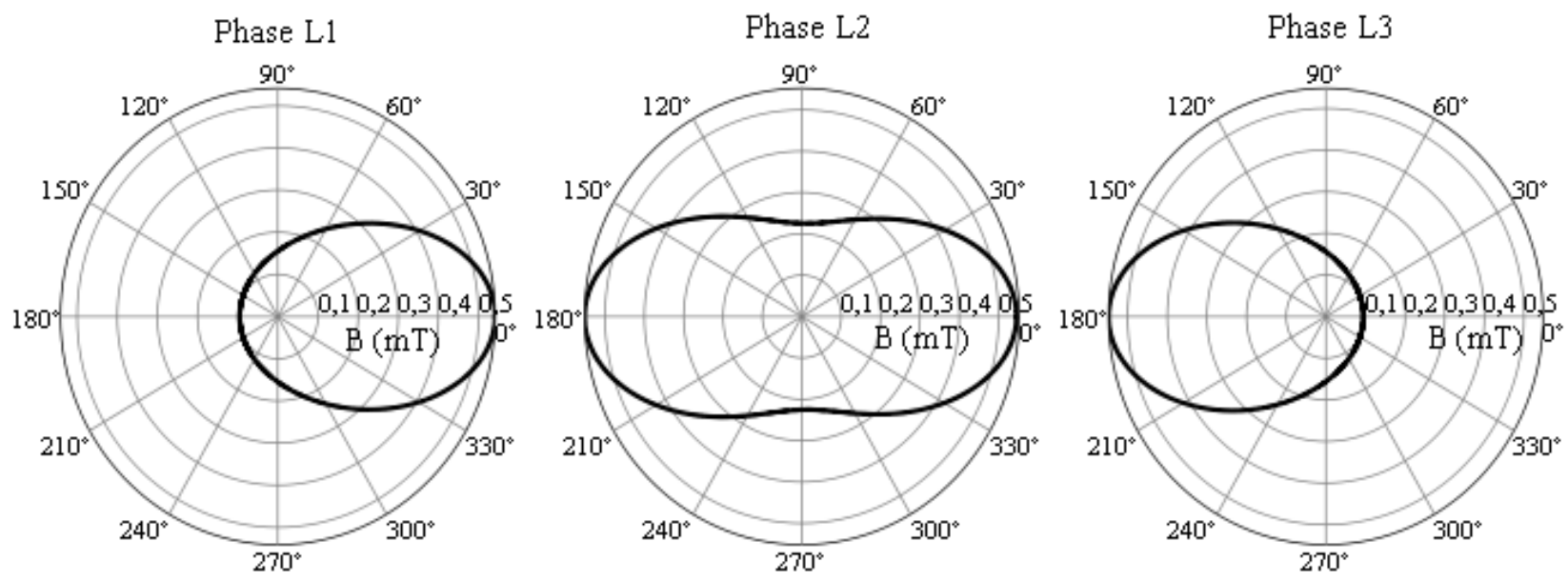

Additionally, Figure 5 provides the RMS values for the total magnetic flux density along the field points of the two above defined observational profiles (located at $0.5 \mathrm{~m}$ and $1 \mathrm{~m}$ above the busduct horizontal symmetry line).

For the purpose of comparison, let us assume for the moment that the screens of the busduct system at hand are grounded on a single end, while at the same time not being mutually bonded on the ungrounded end (nor along the route). This removes the possibility of creating the circulating currents and leaves only the eddy currents in the screens. This analysis would necessitate only a slight rearrangement (i.e., re-computation) of the elements of the matrix Equation (4), particularly in respect to the position of the null-vectors and the all-ones vectors, while the computation procedure for the magnetic field would remain the same as before [12]. 
Figure 5. Distribution of the RMS values of the total magnetic flux density at the field points of the two observational profiles.

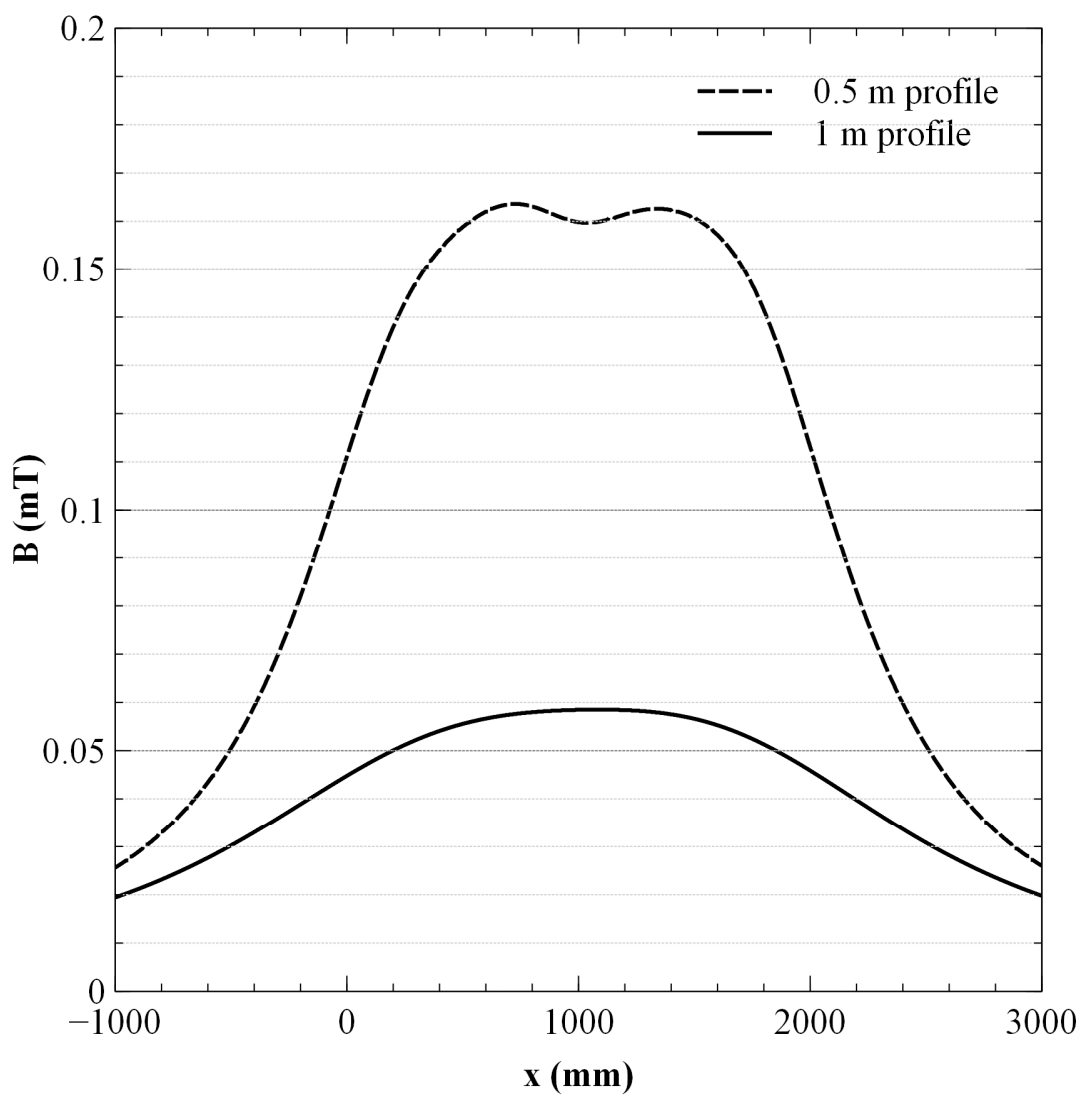

Figure 6. Distribution of the screen currents for both of the above mentioned screen (grounding and bonding) treatments.

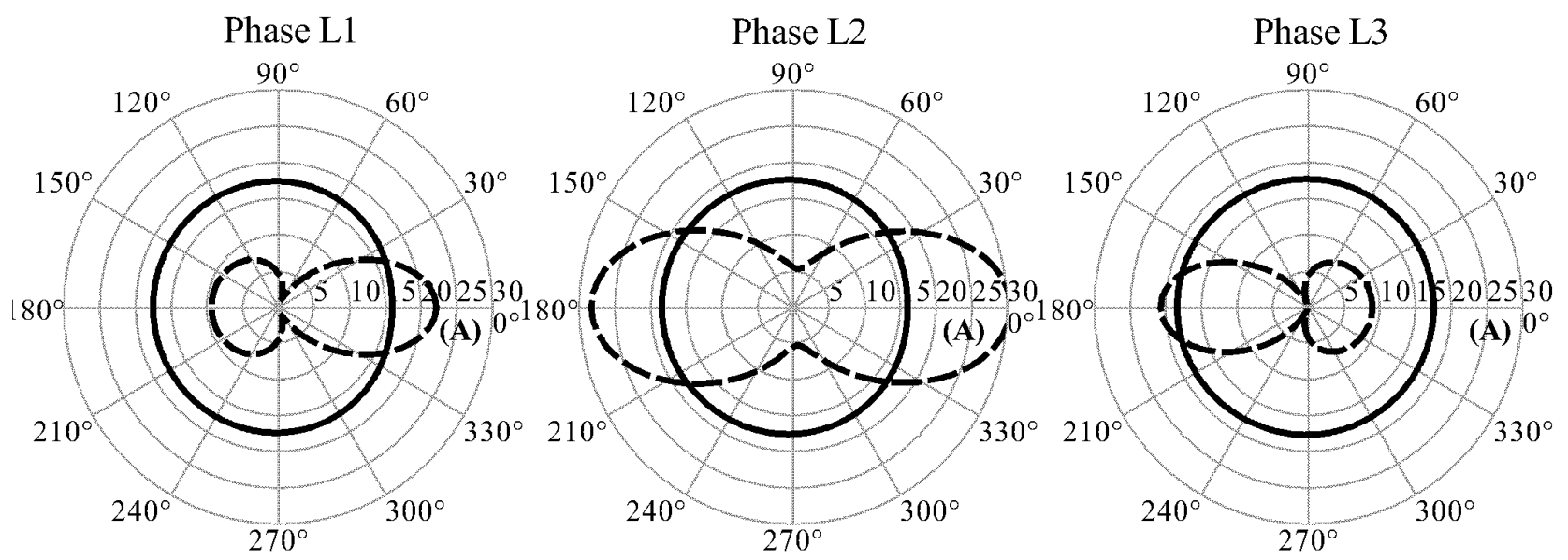

- both-end grounded - - single-end grounded

Figure 6 presents a comparison between the current distributions (RMS current values) in the screens of the high-current busduct system for both of the above mentioned screen (grounding and bonding) treatments. The current distribution in the screens is obtained from the filament currents, depicted in polar coordinates in Figure 6, with the position of the each filament in the figure derived from its actual physical position. It can be appreciated from the Figure 6 that the screen currents 
distribution is highly irregular in the case when the busduct screens are single grounded and while not being mutually bonded on the ungrounded end (nor along the route). This is due to the fact that only eddy current exist, in this instance, in the screens, and as such they circulate along the circumference of each of the screens. It is interesting to note here that the power losses of this busduct system are reduced by some $22 \%$ with this method of screen bonding and grounding. However, at the same time, dangerous voltages could be induced on the ungrounded ends of the busduct, which could possibly endanger personnel's safety at the facility.

Furthermore, Figure 7 presents a comparison between the magnetic field distributions (i.e., total magnetic flux density) along the observational profile located at $1 \mathrm{~m}$ from the symmetry line of the busduct system, obtained for both of the above mentioned screen (grounding and bonding) treatments.

Figure 7. Distribution of total magnetic flux density along the observational profile located $1 \mathrm{~m}$ from the symmetry line of the busduct system for both of the screen treatments.

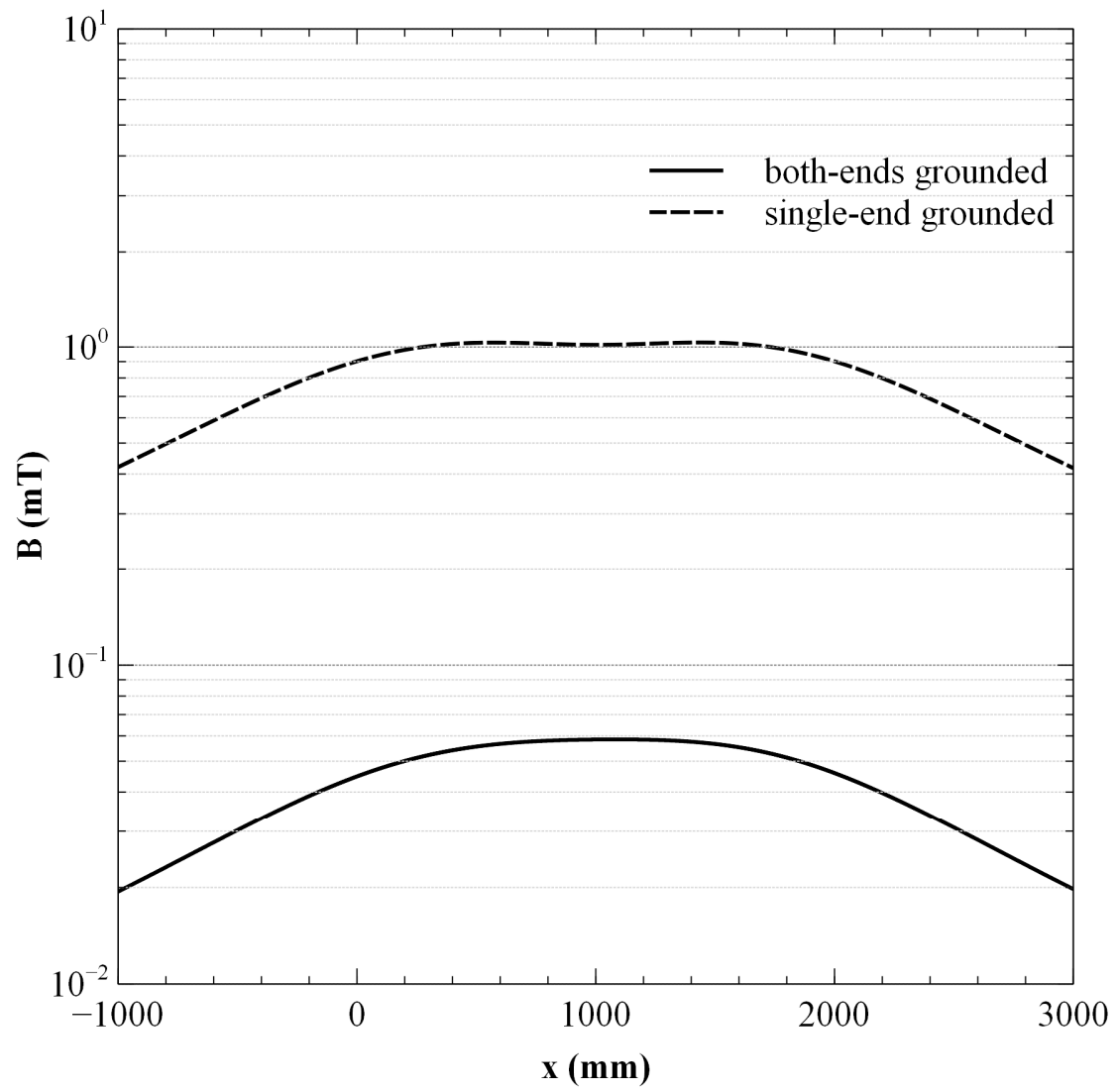

The left coordinate axis in this figure is provided in a logarithmic scale for better visualization and comparison. It can be clearly observed from the Figure 7 that the magnetic flux density is reduced on the order of 17-fold, with the grounding and mutual bonding of the high-current busduct screens. This is true even if the busduct system is grounded on a single end, while its screens are mutually bonded on the ungrounded end. In other words, it is sufficient only to bond (i.e., short-circuit) the busduct screens on its two ends (regardless of the grounding method chosen) to significantly reduce the associated magnetic field. It ought to be mentioned that with the screens being grounded on a single end there is no earth-return current (circulating between grounding systems located on both ends of the busduct route). 
Regarding the above presented results, it could be interesting to note that the magnetic field in the high-current busducts of this type has an elliptical field. In this case the length of the longer semi-axis should be adopted as a magnetic field modulus. Furthermore, magnetic fields in the leftmost and rightmost phases are not symmetrical in relation to the centre phase.

\section{Conclusions}

There are three different influential factors which determine the high-current busduct screen treatment, in terms of its grounding and mutual bonding: (a) total power losses of the busduct system (which produces heating), (b) possible dangerous voltages induced in the screens (i.e., between ungrounded screens and earth), and (c) magnetic fields formed in the vicinity of the busduct system. It is desirable to have very low power losses, very low induced voltages on the screens (that could not endanger personnel safety) and very low magnetic fields (which could not produce adverse effects on the neighboring equipment).

In case of only one high-current busduct system - such as, for example, the one treated in this paper-the lowest power losses would be obtained in case when the screens are grounded on a single end, while not being mutually bonded; there are only eddy currents in the screens in this case. However, at the same time, voltages induced on the ungrounded screen ends (due to the electromotive forces in the screens induced by electromagnetic coupling) could attain dangerous levels, even during normal busduct operation. On the other hand, in case when the screens are either mutually bonded (while still remaining grounded on a single end) or having been grounded on both ends (with or without mutual bonding), the power losses will increase. This is due to the additional formation of circulating currents. However, there are no dangerous voltages on the screens if they are mutually bonded, regardless of the grounding method selected for them (grounded on single end, grounded on both ends or continuously grounded along the route). Furthermore, as has been shown in this paper, associated magnetic field in the vicinity of the high-current busduct system is significantly (i.e., sharply) reduced with the bonding of the busduct screens.

In addition, it is interesting to note that when there are two adjacent parallel busduct systems (e.g., of equal cross-section geometry), the power losses in each of them will be influenced by their mutual distance only in case when the screens are grounded on single end and not bonded on the ungrounded end. Contrary to that, when the screens are mutually bonded, both adjacent and parallel busduct systems behave almost as if they are alone, i.e., their mutual electromagnetic influence is almost completely eliminated. Hence, the unequivocal benefits of the high-current busduct screens bonding provide the reasons supporting the adopted practice, according to which the screens are always mutually bonded; although they could be grounded on a single end (which is sometimes carried out with the generator to step-up transformer connections).

In case of the GIL systems, for the same reasons introduce above, there is a universal practice of mutually bonding the screens and (due to the long distance of the transmission line) their continuous grounding. It is important to emphasize that the environmental impact of this transmission line technology (i.e., GIL) is significantly reduced, compared to the traditional overhead transmission lines of the same capacity, both in terms of the electromagnetic field effects and visual impact on the landscape. Furthermore, due to their low power losses and other afore mentioned benefits, their 
application is certainly expected to increase in the near future (e.g., in offshore wind farm applications and for bringing the bulk transmission power to major city centers) $[25,26]$.

In the end, it could be stated that the here presented mathematical model for the computation of the high-current busduct or GIL system current distribution and electromagnetic fields could be easily extended to the cases of two (or even more) adjacent and parallel systems (with equal or different cross-section geometry). Moreover, different geometry of the busduct phase conductors could be treated as well (e.g., octagonal phase conductors, U-profiles, flat rectangular conductors, etc.).

\section{References}

1. ABB. Generator Busduct, Power Technology Systems; ABB AG: Mannheim, Germany, 2009. Available online: http://www.abb.com (accessed on 12 October 2011).

2. GE Energy. Segregated and Non-Segregated Bus Ducts, GE, St-Augustine, Quebec, Canada. Available online: http://www.genergy.com/prod_serv/products/bus_ducts/en/seg_and_non_seg.htm (accessed on 12 October 2011).

3. Končar. Isolated Phase Busbar Solutions, Končar-Metal Constructions, Zagreb, Croatia. Available online: http://www.koncar-mk.hr/kmk_isolated_phase_busbars_[3][en].htm (accessed on 12 October 2011).

4. Siemens. Gas-Insulated Transmission Lines (GIL); High-Power Transmission Technology, Siemens AG, Energy Sector: Erlangen, Germany, 2010.

5. Kunze, D.; Binder, E.; Türk, J.; Pöhler, S.; Alter, J. Gas-Insulated Transmission LinesUnderground Power Transmission Achieving a Maximum of Operational Safety and Reliability. In Proceedings of the International Conference on Insulated Power Cables (JICABLE '07), Paris-Versailles, France, 24-28 June 2007.

6. Koch, J. Experience with 2nd Generation Gas-Insulated Transmission Lines GIL. In Proceedings of the International Conference on Insulated Power Cables (JICABLE '03), Paris-Versailles, France, 22-26 June 2003; Paper A.3.2.

7. Drews, A.; Rathke, C.; Siebert, M.; Heinemann, D.; Hühnerbein, B.; Hofmann, L.; Koch, H.; Kunze, H.; Hoppensack, H.-J.; Lohrenscheit, H.; et al. Network of offshore wind farms connected by gas insulated transmission lines? Available online: http://www.forwind.de/forwind/files/ ewec2008_gil3-1.pdf (accessed on 12 October 2011).

8. Benato, R. Gas Insulated Transmission Lines in Railway Galleries. IEEE Trans. Power Deliv. 2005, 20, 704-709.

9. Menke, P. Innovative Solutions for Bulk Power Transmission; Siemens Energy Sector, Siemens AG: Erlangen, Germany, 2011.

10. Koch, H.; Schuette, A. Gas insulated transmission lines for high power transmission over long distances. Electric Power Syst. Res. 1998, 44, 69-74.

11. Alberta Energy. Assessment and Analysis of the State-of-the-Art Electric Transmission Systems with Specific Focus on High-Voltage Direct Current (HVDC), Underground or Other New or Developing Technologies; Alberta Energy: Stantec, Canada, 2009.

12. Sarajčev, P.; Goić, R. Power loss computation in high-current generator bus ducts of rectangular cross-section. Electric Power Compon. Syst. 2010, 38, 1469-1485. 
13. Piatek, Z. Total Eddy Currents Induced in Screens of a Symmetrical Three-Phase Single-Pole Gas-Insulated Transmission Line (GIL). Available online: http://advances.utc.sk/index.php/AEEE/ article/download/94/147 (accessed on 12 October 2011).

14. Benato, R.; Dughiero, F.; Forzan, M.; Paolucci, A. Proximity effect and magnetic field calculation in GIL and in isolated phase bus ducts. IEEE Trans. Magn. 2002, 38, 781-784.

15. Infolytica. MagNet v7: 2D/3D Electromagnetic Field Simulation Software. Available online: http://www.infolytica.com/en/products/magnet/ (accessed on 12 October 2011).

16. Piatek, Z.; Kusiak, D.; Szczegielniak, T. Electromagnetic Field and Impedances of High Current Busducts. In Proceedings of the International Symposium Modern Electric Power Systems (MEPS), Wroclaw, Poland, 20-22 September 2010.

17. Piatek, Z.; Kusiak, D.; Szczegielniak, T. Influence of the screen on the magnetic field of the flat three phase high current busduct. Prz. Elektrotechniczny 2010, 86, 89-91.

18. Kovač, N.; Sarajčev, I.; Poljak, D. Nonlinear-coupled electric-thermal modeling of underground cable systems. IEEE Trans. Power Deliv. 2006, 21, 4-14.

19. Kovač, N.; Sarajčev, I.; Poljak, D. Nonuniformity modeling of a particular aluminum sheath temperatures and losses. IEE Proc. Gener. Transm. Distrib. 2006, 153, 553-559.

20. Carson, J.R. Wave propagation in overhead wires with ground return. Bell Syst. Tech. J. 1926, 5, $539-554$.

21. Dommel, H.W. EMTP Theory Book; Microtran Power System Analysis Corporation: Vancouver, Canada, 1992.

22. Sarajcev, I. Power loss computation of cable systems. Ph.D. Dissertation, University of Zagreb, FER, Zagreb, Croatia, 1985 (in Croatian).

23. Surutka, J. Electromagnetics, 2nd ed.; Engineering Books: Belgrade, Yugoslavia, 1966 (in Serbian).

24. Sarajčev, P. Analysis of Electromagnetic Influences between Two Adjacent Generator High-Current Busducts in HPP Zakucac; University of Split: Split, Croatia, 2011 (in Croatian).

25. Koch, H.; Benato, R.; Laußegger, M.; Köhler, M.; Leung, K.K.; Mirebeau, P.; Kindersberger, J.; Kunze, D.; di Mario, C.; Renaud, F.; Bowmann, G. Application of long high capacity gas-insulated lines in structures. IEEE Trans. Power Deliv. 2007, 22, 619-626.

26. Awad, R.; Peacock, C.; Benato, R.; Butt, I.; de Wild, F.; Takahashi, Y.; Jeyapalan, J.K.; Kim, J.T.; Monteys, J.; Moreau, C.; Oberger, K.; Sakuma, S.; Woschitz, R.; Yoon, K.T. Cable Systems in Multi Purpose or Shared Structures; CIGRÉ Technical Brochure N 403 , Working Group B1.08; CIGRÉ: Paris, France, February 2010.

(C) 2011 by the authors; licensee MDPI, Basel, Switzerland. This article is an open access article distributed under the terms and conditions of the Creative Commons Attribution license (http://creativecommons.org/licenses/by/3.0/). 